\title{
Comparison of dexmedetomidine and ketamine for the analgesic effect using intravenous patient-controlled analgesia after gynecological abdominal surgery
}

\author{
Wonjin Lee, Jung Dae Shin, Kwangrae Cho, and Myoung-Hun Kim \\ Department of Anesthesiology and Pain Medicine, Busan Paik Hospital, Collage of Medicine, Inje University, Busan, Korea
}

Intravenous patient-controlled analgesia (IV-PCA) using opioids allows the patient to control the amount of analgesic according to the degree of pain. For effective pain reduction and avoidance of opioid-related complications, studies have investigated methods of combining opioid use with drugs such as NSAIDs, ketamine, clonidine, and dexmedetomidine [1]. The highly selective $\alpha_{2}$-agonist dexmedetomidine is effective in sedating and reducing anxiety. In addition, because it retains a simultaneous analgesic effect, it is expected to be helpful in postoperative pain management [2]. The authors compared ketamine and dexmedetomidine, each in combination with fentanyl, in postoperative IV-PCA for patients scheduled for gynecological abdominal surgery. For each method, we examined the reduction in pain score and opioid consumption as well as whether it was effective in reducing opioid-related complications.

After obtaining approval from the Institutional Review Board, and informed consent was obtained. Ninety patients (20 to 60 years, American Society of Anesthesiologists [ASA] physical status I-II) scheduled for gynecological abdominal surgery were divided into three groups: a control group (Group C: $\mathrm{n}$ $=30$ ), a ketamine group (Group $\mathrm{K}: \mathrm{n}=30$ ), and a dexmedetomidine group (Group D: $\mathrm{n}=30$ ). Near the end of surgery, all three groups were injected with fentanyl $0.5 \mu \mathrm{g} / \mathrm{kg}$, ketorolac $30 \mathrm{mg}$, and ramosetron $0.3 \mathrm{mg}$ for postoperative pain management. PCA was performed using IV-PCA equipment (AutoMed $3000^{\circledR}$, Ace Medical, Seoul, Korea). The pharmacological com- binations for the three groups were as follows: fentanyl $20 \mu \mathrm{g} / \mathrm{kg}$, ketorolac $180 \mathrm{mg}$, and ramosetron $0.6 \mathrm{mg}$ for all three groups; normal saline for Group C; normal saline plus ketamine $40 \mathrm{mg}$ for Group K; and normal saline plus dexmedetomidine (Prece$\operatorname{dex}^{\circledR}$, Hospira, Rocky Mount, NC, USA) $500 \mu \mathrm{g}$ for Group D. Each mixture was made in $100 \mathrm{ml}$ solutions. The basal infusion rate was set at $2 \mathrm{ml} / \mathrm{hr}$, the bolus at $2 \mathrm{ml}$, and the lockout time at 10 minutes. After the completion of surgery, patients were transferred to the ward. The IV-PCA consumption volume, pain score (VAS; visual analogue scale) and side effects were assessed at postoperative hours $1,6,12,24,36$, and 48 . When the patients required pain control, ketorolac $30 \mathrm{mg}$ or tramadol $50 \mathrm{mg}$ was administered. To treat nausea and vomiting, an IV injection of macperan $10 \mathrm{mg}$ or ondansetron $4 \mathrm{mg}$ was administered. If nausea and vomiting continued despite the administration of antiemetics or if the sedation score was more than 2, the IV-PCA background infusion rate and bolus were reduced by $0.4 \mathrm{ml}$, respectively. If respiration was inhibited (fewer than 8 breaths/ min), naloxone 0.1-0.4 mg was administered, and IV-PCA was discontinued until the respiration rate reached 9 breaths/min or higher. Data were analyzed using SAS 9.2 (SAS Institute Inc, Cary, NC, USA). One-way ANOVA was performed for the demographic data, and repeated measures ANOVA was performed for the between-group comparison of VAS pain scores, accumulated consumption of IV-PCA, accumulated consumption using bolus, and patient information. A P value of 0.05 or lower was considered statistically significant.

Corresponding author: Wonjin Lee, M.D., Department of Anesthesiology and Pain Medicine, Busan Paik Hospital, Collage of Medicine, Inje University, 633-165, Gaegum-dong, Jin-gu, Busan 614-735, Korea. Tel: 82-51-890-6520, Fax: 82-51-898-4216, E-mail: 2wonjin@hanmail.net (c) This is an open-access article distributed under the terms of the Creative Commons Attribution Non-Commercial License (http:// creativecommons.org/licenses/by-nc/3.0/), which permits unrestricted non-commercial use, distribution, and reproduction in any medium, provided the original work is properly cited. 
A



C

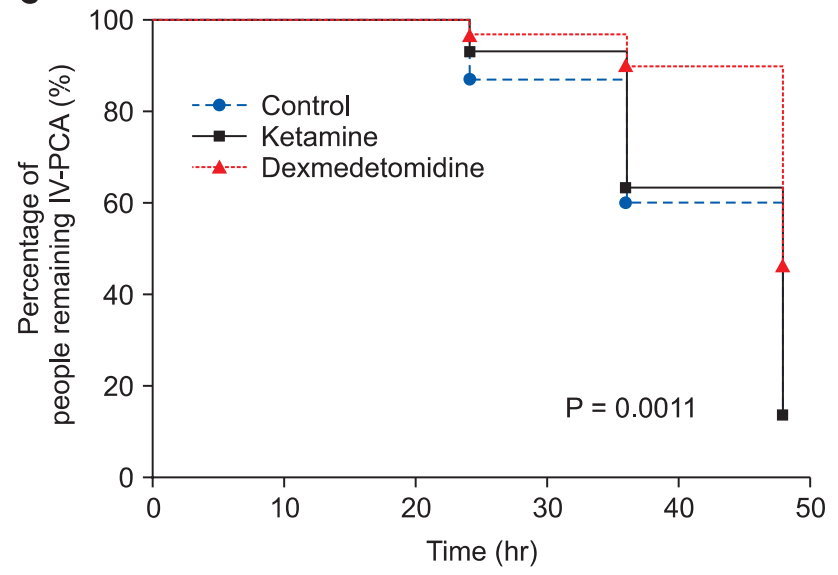

There were no significant differences between the three groups in age, body mass index, ASA class, type of surgery, or surgery time. In Group K, the pain score was lower (in a resting state: 24 and 36 hours after surgery; during movement: 6, 12, 24, 36 , and 48 hours after surgery) and there was no significant difference in IV-PCA consumption volume compared with Group C. In Group D, the pain score was significantly lower (in a resting state: 24 and 36 hours after surgery; during movement: 6 , 12, 24, 36, and 48 hours after surgery) (Figs. 1A and 1B) and the total IV-PCA consumption volume was lower (26\% at 24 hours, $10 \%$ at 48 hours) compared with Group C. When Group K and Group D were compared, there were no significant differences in the VAS score in a resting state or during movement. In addition, there were no significant differences in accumulated total consumption and bolus consumption between Group $\mathrm{C}$ and Group $\mathrm{K}$. The number of patients that used the entire IV-PCA volume of $100 \mathrm{ml}$ at 24, 36, and 48 hours after surgery was highest in Group C, followed by Group K and Group D, respectively (Fig. 1C). In order of descending frequency, complications (in groups $\mathrm{C}, \mathrm{K}, \mathrm{D})$ that occurred were nausea $(16,14,14)$ vomiting $(4,1,0)$, sedation $(12,13,15)$, dizziness $(2,8,2)$, pruritus $(0,0,2)$, and re-

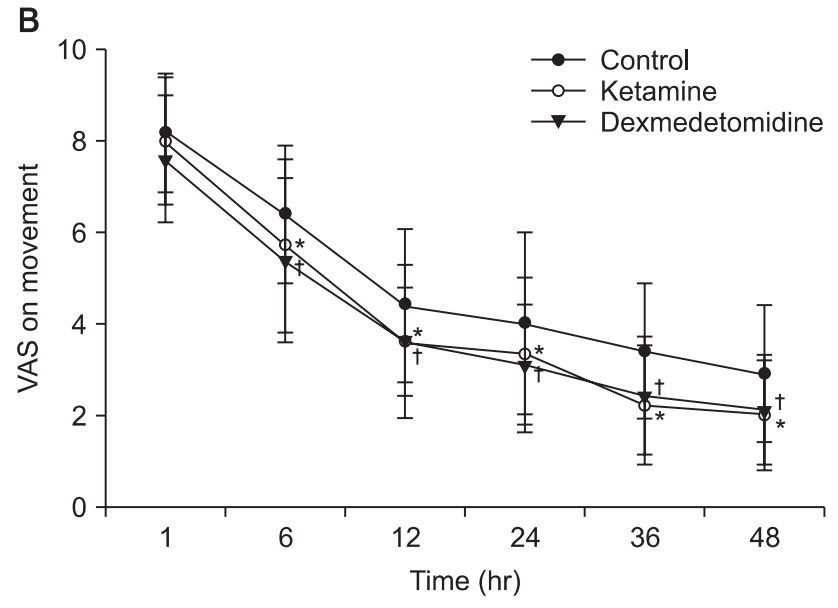

Fig. 1. (A) Visual analogue scale (VAS) pain score at rest during the 48hour study, (B) Visual analogue scale (VAS) pain score on movement during the 48 -hour study. Values are mean $\pm \mathrm{SD}$. $* \mathrm{P}<0.05$ (group K versus group C), ${ }^{\dagger} \mathrm{P}<0.05$ (group D versus group C), (C) Kaplan-Meier graph shows the time it takes to consume all IV-PCA, in each group. Values are expressed as percentages. There were significant differences among the three groups. Graphs were compared with the log-rank test $(\mathrm{P}=0.0011$ among the three groups, $\mathrm{P}=0.0015$ group $\mathrm{C}$ versus group $\mathrm{D}$, $\mathrm{P}=0.0008$ group $\mathrm{K}$ versus group $\mathrm{D}$ ).

spiratory depression $(0,1,0)$. Dizziness was significantly higher in Group $\mathrm{K}$ than in the other groups.

The mechanism of the analgesic effect of dexmedetomidine as an $\alpha_{2}$-agonist is not yet completely understood, but it is thought that the spinal cord is the most important area. The $\alpha_{2}-$ adrenergic receptors of the substantia gelatinosa are activated in the dorsal horn of the spinal cord; this represses the neurotransmission of peripheral $\mathrm{A} \delta$ and $\mathrm{C}$ fiber, represses wide dynamic range neurons, stimulates the secretion of acetylcholine, stimulates the serotonergic system, and represses the secretion of substance P, resulting in an analgesic effect [3-5]. Lin et al. [1], who conducted a study with patients receiving total abdominal hysterectomy, reported that combined morphine and dexmedetomidine significantly reduced the pain score over 24 hours of observation compared with a group that received only morphine. Furthermore, there was a reduction in morphine consumption of approximately $29 \%$. These findings are similar to ours; in our study, the pain score was significantly reduced and there was an approximately $26 \%$ reduction in PCA consumption in IV-PCA up to 24 hours after surgery compared with Group C. Our study also showed a higher frequency of dizziness in 
Group K compared with Group C and Group D. There were no significant differences in sedation, hypoventilation, and itching between each group. In conclusion, low-dose ketamine added to IV-PCA in gynecological abdominal surgery did not decrease total IV-PCA consumption but reduced the pain score relative to Group C, while dexmedetomidine reduced both total IV-PCA consumption and the pain score relative to Group C.

\section{References}

1. Lin TF, Yeh YC, Lin FS, Wang YP, Lin CJ, Sun WZ, et al. Effect of combining dexmedetomidine and morphine for intravenous patientcontrolled analgesia. Br J Anaesth 2009; 102: 117-22.

2. Sadhasivam S, Boat A, Mahmoud M. Comparison of patient-controlled analgesia with and without dexmedetomidine following spine surgery in children. J Clin Anesth 2009; 21: 493-501.

3. Paris A, Tonner PH. Dexmedetomidine in anaesthesia. Curr Opin Anaesthesiol 2005; 18: 412-8.

4. Correa-Sales C, Rabin BC, Maze M. A hypnotic response to dexmedetomidine, an alpha 2 agonist, is mediated in the locus coeruleus in rats. Anesthesiology 1992; 76: 948-52.

5. Gaumann DM, Brunet PC, Jirounek P. Clonidine enhances the effects of lidocaine on C-fiber action potential. Anesth Analg 1992; 74: 719-25. 